TRANSACTIONS OF THE

AMERICAN MATHEMATICAL SOCIETY

Volume 349, Number 11, November 1997, Pages 4659-4667

S 0002-9947(97)01897-7

\title{
COHEN-MACAULAY SECTION RINGS
}

\author{
ZHOU CAIJUN
}

\begin{abstract}
In this paper, we study the section rings of sheaves of CohenMacaulay algebras (over a field $F$ ) on a ranked poset. A necessary and sufficient condition for these rings to be Cohen-Macaulay will be given. This is a further generalization of a result of Yuzvinsky, which generalizes Reisner's theorem concerning Stanley-Reisner rings.
\end{abstract}

\section{INTRODUCTION}

Throughout this paper, every ring is associative, commutative, Noetherian and has an identity element 1 . Every ring homomorphism maps 1 to 1 . If $J$ is an ideal of a ring $R$, we use the convention

$$
\operatorname{bight}(J)=\max \{h t(P) \mid P \text { is a minimal prime of } J\} .
$$

Let $X$ be a finite poset with its natural increasing order topology and $\mathfrak{A}$ a sheaf of rings on $X$. The algebraic structure of the section ring $\Gamma(\mathfrak{A})$ is sometimes related to the topological structure of $X$. One interesting thing is that a Stanley-Reisner ring can be viewed as the section ring of a sharp flasque sheaf of regular algebras on an atomic prelattice. Under this observation, Yuzvinsky studied the section rings of sharp flasque sheaves of regular algebras (over a field $F$ ) on an atomic prelattice, and obtained a necessary and sufficient condition for such rings to be Cohen-Macaulay (abbr. CM) [8, Theorem 6.4]. This generalizes Reisner's theorem, first proved in [6], concerning the $C M$ property of Stanley-Reisner rings.

Now, before we state the main result of the paper, let us recall some notions and notations which will be used throughout without further explanations. All other unexplained notions and notations are standard and can be found in [4], [5].

Let $X$ be a finite poset. We denote by $\operatorname{rk}(X)$ the number of elements of a longest chain in $X$. A ranked poset is a finite poset in which every maximal chain has the same length. For $z \in X$, we always put $X_{z}=\{x \in X \mid x>z\}, \bar{X}_{z}=\{x \in X \mid x \geq$ $z\}, X^{z}=\{x \in X \mid x<z\}, \bar{X}^{z}=\{x \in X \mid x \leq z\}$. We shall use the convention that $\operatorname{rk}(z)=\operatorname{rk}\left(\bar{X}^{z}\right)$, and $X_{\min }=\{x \in X \mid x$ is a minimal element $\}$. Moreover, the collection of all the chains forms an abstract simplicial complex. It is clear that the dimension of this complex is $\operatorname{rk}(X)-1$. We denote by $F$ a fixed arbitrary field and by $\widetilde{H}^{i}(X, F)$ the usual $i$ th reduced cohomology group of the above abstract complex. A poset $X$ is said to be $F$-spherical if for every $z \in X$, the reduced cohomology groups $\widetilde{H}^{i}\left(X^{z}, F\right)=0$ for $0 \leq i \leq \operatorname{rk}(z)-2$. The poset $X$ is said to be $F$-acyclic if $\widetilde{H}^{i}(X, F)=0$ for $i \geq 0$.

Received by the editors May 18, 1996.

1991 Mathematics Subject Classification. Primary 13C14, 13H10, 06A07.

(C)1997 American Mathematical Society 
Let $X$ be a poset. The natural increasing order topology on $X$ is defined as the topology in which a subset $Y$ is open if and only if $x \geq z \in Y$ implies $x \in Y$. The general definition of a sheaf on a topological space (see, e.g., [4]) can be easily specified for the order topology. For example, a presheaf $\mathfrak{A}=\left(A_{x}, \rho_{y x}\right)$ of rings on a basis of the increasing order topology of $X$ consists of rings $(\mathfrak{A})_{x}=A_{x}$ for $x \in X$ which are called stalks of $\mathfrak{A}$, and ring homomorphisms $\rho_{y x}: A_{x} \rightarrow A_{y}$ for every $x, y \in X$ such that $x \leq y$. The homomorphisms should satisfy the following conditions:

(i) $\rho_{x x}=1_{A_{x}}$ for every $\quad x \in X$, and

(ii) $\rho_{z y} \rho_{y x}=\rho_{z y}$ for every $\quad x, y, z \in X \quad$ with $\quad x \leq y \leq z$.

We will use the same notation $\mathfrak{A}=\left(A_{x}, \rho_{y x}\right)$ to denote the sheaf yielded by the unique sheafification of the presheaf $\mathfrak{A}=\left(A_{x}, \rho_{y x}\right)$.

Let $\mathfrak{A}=\left(A_{x}, \rho_{y x}\right)$ be a sheaf of rings on a finite poset $X$. It is easy to see that the section ring of $\mathfrak{A}$ is $\Gamma(\mathfrak{A})=\left\{s \in \prod_{x \in X} A_{x} \mid \rho_{y x}(s(x))=s(y), x, y \in X, x \leq y\right\}$, where $s(x)$ is the image of $s$ under the natural projection of $\prod_{y \in X} A_{y}$ to $A_{x}$. Clearly, $R=\Gamma(\mathfrak{A})$ is a subring of $\prod_{x \in X} A_{x}$, and $\Gamma\left(\mathfrak{A} \mid \bar{X}_{x}\right)=A_{x}$. One can prove easily that $R$ is Noetherian. For every nonempty subset $Y \subseteq X$, we denote by $\rho_{Y}$ the restriction homomorphism $R \rightarrow \Gamma(\mathfrak{A} \mid Y)$, and by convention we set $\rho_{x}=\rho_{\bar{X}_{x}}$ for $x \in X$. Also we always put $J_{x}=\operatorname{Ker} \rho_{x}=\{s \in \Gamma(\mathfrak{A}) \mid s(x)=0\}$. Clearly, $J_{x}$ is an ideal of $R, J_{x} \subset J_{y}$ for $x \leq y$, and $\bigcap_{x \in X_{\min }} J_{x}=0$. We say that $\mathfrak{A}$ is sharp if $\operatorname{ht}\left(\operatorname{Ker}\left(\rho_{y x}\right)\right) \neq 0$ in $A_{x}$ for every $x<y$. When $A_{x}$ is a domain for each $x \in X$, this definition agrees with the definition in [8]. The sheaf $\mathfrak{A}$ is called flasque if $\rho_{U}$ is an epimorphism for every nonempty open subset $U$. In particular, if $\mathfrak{A}$ is flasque all homomorphisms $\rho_{x}, \rho_{y x}$ are epimorphisms. In this case, we have $A_{x} \simeq R / J_{x}$. If $\mathfrak{A}$ is sharp flasque, it is easy to see that no minimal prime ideal of $J_{x}$ can be a minimal prime ideal of $J_{y}$ for $x<y$. This fact will play an important role in the proof of our main result when we localize the sheaf $\mathfrak{A}$ at a prime $P$ of $R$.

In this paper, our main goal is to prove the following result, which generalizes the main theorem of [8], in which the algebras $A_{x}$ are assumed to be regular domains and the poset is assumed to be an atomic prelattice.

Theorem 2.4. Let $X$ be a ranked poset and $\mathfrak{A}=\left(A_{x}, \rho_{y x}\right)$ be a sharp flasque sheaf of $C M F$-algebras on $X$. Put $R=\Gamma(\mathfrak{A})$. Then the following conditions are equivalent:

(i) $R$ is $C M$;

(ii) $X$ is $F$-spherical, and $X^{z}$ is $F$-acyclic for every $z \in X$ with $\operatorname{bight}\left(J_{z}\right)>$ $\operatorname{rk}(z)-1$.

\section{Section Rings of Flasque Sheaves of $C M$ Local Rings}

In [8], Yuzvinsky studied the basic properties of sheaves of integral domains on a finite poset. In this section, we obtain similar results for the sheaves of $C M$ local rings on a finite poset. So in the propositions below $\mathfrak{A}=\left(A_{x}, \rho_{y x}\right)$ is a fixed sharp flasque sheaf of $C M$ local rings on a finite poset $X$, and $R=\Gamma(\mathfrak{A})$.

Proposition 1.1. Let $x, y$ be two elements of $X$. If $J_{x}$ is contained in some minimal prime ideal of $J_{y}$, then $x \leq y$.

Proof. Put $Y=\bar{X}_{x} \cup \bar{X}_{y}$. Let $z_{1}, \ldots, z_{k}$ be the successors of $y$ and set $K_{i}=$ $\operatorname{Ker} \rho_{z_{i} y}(1 \leqslant i \leqslant k)$. We have $K_{i} \simeq J_{z_{i}} / J_{y}$. Since $\mathfrak{A}$ is sharp, we get that ht $\left(J_{z_{i}} / J_{y}\right)>0$ in the ring $R / J_{y}$, which means that ht $\left(\bigcap_{i=1}^{k} K_{i}\right)>0$. As $A_{y}$ is 
$C M$, there exists a section $t \in \Gamma(\mathfrak{A} \mid Y)$ such that $t(y) \in \bigcap_{i=1}^{k} K_{i}$ is an $A_{y}$-regular element and $t(u)=0$ for $u \in Y \backslash\{y\}$. Extend $t$ to a section $\bar{t} \in R$. Clearly, $\bar{t} \in J_{x}$ and $\bar{t}$ cannot be an element of each minimal prime ideal over $J_{y}$.

Proposition 1.2. The set of minimal prime ideals of $R$ is equal to $\bigcup_{x \in X_{\text {min }}}\{P \mid$ $P$ is a minimal prime of $\left.J_{x}\right\}$.

Proof. Clearly, $\bigcap_{\mathrm{rk}(x)=1} J_{x}=0$. So for an arbitrary minimal prime ideal $P$ of $R$, we have $P \supset \bigcap_{\text {rk }(x)=1} J_{x}$. This shows that $P \supseteq J_{x_{0}}$ for some $x_{0} \in X_{\min }$, and $P$ is a minimal prime ideal of $J_{x_{0}}$.

Conversely, for $x \in X_{\min }$, let $P$ be a minimal prime ideal of $J_{x}$. If $P$ is not a minimal prime ideal of $R$, then there exists a minimal prime ideal $P^{\prime}$ such that $P \supset P^{\prime}$ and $P^{\prime} \nsupseteq J_{x}$. On the other hand, $P^{\prime} \supseteq J_{y}$ for some $y \in X_{\min }$ and $y \neq x$. Hence $P \supseteq P^{\prime} \supseteq J_{y}$, by Proposition 1.1, which is a contradiction. So $P$ is a minimal prime ideal of $R$.

Proposition 1.3. If $X$ is a ranked poset with a unique maximal element and $R$ is a local $C M$ ring, then there exists an $R$-sequence $r_{1}, \ldots, r_{n-1}$ such that $r_{1}(x)=$ $\cdots=r_{i-1}(x)=0$ for every element $x \in X$ with $\mathrm{rk}(x)=i \geqslant 2$. Furthermore, if ht $J_{x}=i-1$ for some $x \in X(\operatorname{rk}(x)=i)$, then $r_{i}(x), \ldots, r_{n-1}(x)$ is an $A_{x}$-sequence.

Proof. First, we construct elements $r_{i}(1 \leqslant i \leqslant n-1)$. For every $i(1 \leqslant i \leqslant n)$, we put $J(i)=\bigcap_{\mathrm{rk}}(x)=i J_{x}$. Since $R$ and $A_{x}$ (for every $\left.x \in X\right)$ are $C M$ local rings, we have ht $J_{x}+\operatorname{dim} A_{x}=\operatorname{dim} R$ for every $x \in X$. Now we claim

$$
0=\text { ht } J(1)<\text { ht } J(2)<\cdots<\text { ht } J(n) .
$$

In fact, if there exists $i_{0}\left(0 \leqslant i_{0}<n\right)$ such that ht $J\left(i_{0}\right)=$ ht $J\left(i_{0}+1\right)$, then there exists a minimal prime ideal $P$ over $J\left(i_{0}+1\right)$ which is also a minimal prime ideal over $J(i)$. Put $Y_{i_{0}}=\left\{x \in X \mid \operatorname{rk}(x) \geqslant i_{0}\right\}$ and $Y_{i_{0}+1}=\left\{x \in X \mid \operatorname{rk}(x) \geqslant i_{0}+1\right\}$. Since $\mathfrak{A}$ is flasque, $R / J(i)$ and $R / J(i+1)$ are the rings of sections of the flasque sheaves $\mathfrak{A} \mid Y_{i_{0}}$ and $\mathfrak{A} \mid Y_{i_{0}+1}$, respectively. Hence according to Proposition 1.2, $P$ must be a minimal prime ideal for some $J_{x}$ and $J_{y}$, where $\operatorname{rk}(x)=i_{0}$, rk $(y)=i_{0}+1$. It follows from Proposition 1.1 that $x<y$, so the sharp property implies ht $J_{x}<$ ht $J_{y}$. On the other hand, $R, A_{x}, A_{y}$ are all $C M$ local rings, so $J_{x}$ and $J_{y}$ must be unmixed ideals. This shows that ht $J_{x}=$ ht $J_{y}$, which is a contradiction.

Hence we can choose an $R$-sequence $\left(r_{1}, \ldots, r_{n-1}\right)$ such that $r_{i} \in J(i+1) \backslash J(i)$. This completes the construction of the elements $r_{i}$.

The second assertion follows easily from $C M$ properties.

\section{Main Result}

In this section, we discuss our main result. First of all, we quote some important facts from [3], [8] and [9], which will be used several times in the proof of the main result.

Lemma 2.1 (cf. [8, Section 4,(4.1)]). Let $X$ be a ranked poset and $\mathfrak{A}=\left(A_{x}, \rho_{y x}\right)$ be a sheaf of F-algebras on $X$. If $X$ is $F$-spherical, then the sheaf cohomology groups $H^{i}(X, \mathfrak{A})$ can be computed as homology groups of the following complex of $\Gamma(\mathfrak{A})$-modules:

$$
\begin{gathered}
0 \longrightarrow C^{0} \stackrel{\partial_{0}}{\longrightarrow} \ldots \stackrel{\partial_{r-2}}{\longrightarrow} C^{n-1} \longrightarrow 0 \\
\text { where } C^{i}=\bigoplus_{\operatorname{rk}(x)=i+1} \widetilde{H}^{i-1}\left(X^{x}, A_{x}\right), i \geqslant 1, n=\operatorname{rk}(X) \text {, and } C^{0}=\bigoplus_{\operatorname{rk}(x)=1} A_{x} .
\end{gathered}
$$


Remark. In Lemma 2.1, since every $A_{x}(x \in X)$ is an algebra over the field $F$, it follows from the Universal Coefficient Theorem that each $C^{i}$ is the direct sum of $A_{x}$ with $\operatorname{rk}(x)=i+1$, taken each with some multiplicity. In particular, if $X^{x}$ is $F$-acyclic, then $A_{x}$ is not a summand of $C^{i}$.

Let $X$ be a poset and $\mathfrak{A}=\left(A_{x}, \rho_{y x}\right)$ a sheaf of rings on $X$. Set $R=\Gamma(\mathfrak{A})$ and let $P$ be a prime ideal of $R$. Put $Y=\left\{x \in X \mid J_{x} \subseteq P\right\}$. Note that $Y \neq \emptyset$. The localization of $A_{x}$ and $\rho_{y x}$ at $P$ forms a sheaf $\mathfrak{A}_{P}$ of local rings on $Y$.

Lemma 2.2 ([9, Proposition 3.1]). $R_{P} \simeq \Gamma\left(\mathfrak{A}_{P}\right) . \mathfrak{A}$ is flasque if and only if $\mathfrak{A}_{P}$ is flasque for every prime ideal $P$ of $R$.

Due to Proposition 1.1 and Lemma 2.2, we can prove the following proposition word for word as Proposition 6.1 in [8].

Proposition 2.3. Let $X$ be a poset and $\mathfrak{A}$ a sharp flasque sheaf of $C M$ local rings on $X$. If $z \in X$ is such that $X^{z}$ is not connected, then $\operatorname{depth}_{J_{z}}(R) \leq 1$.

Now we are ready for the main result.

Theorem 2.4. Let $X$ be a ranked poset and $\mathfrak{A}=\left(A_{x}, \rho_{y x}\right)$ a sharp flasque sheaf of $C M F$-algebras on $X$. Put $R=\Gamma(\mathfrak{A})$. Then the following conditions are equivalent:

(i) $R$ is $C M$;

(ii) $X$ is F-spherical, and $X^{z}$ is F-acyclic for every $z \in X$ with bight $J_{z}>$ $\operatorname{rk}(z)-1$.

Proof. First we make some reductions. Since $\mathfrak{A}$ is sharp and flasque, the localization $\mathfrak{A}_{P}$ of $\mathfrak{A}$ at $P$ is also a sharp sheaf on $Y=\left\{z \in X \mid J_{z} \subseteq P\right\}$. Thus, by Lemma $2.2, \mathfrak{A}_{P}$ is a sharp flasque sheaf of $C M$ local $F$-algebras on $Y$ with the section ring $R_{P}$. It is clear that $Y$ is a closed subposet of $X$. We observe that $Y$ has a unique maximal element. Suppose on the contrary that we can choose two elements $z_{1}, z_{2}$ that are maximal in $Y$. Put $U=\left\{z_{1}, z_{2}\right\}$. It is clear that $U$ is open in $Y$. Take an arbitrary element $s \in R_{P}$. Since $\mathfrak{A}_{P}$ is flasque, there exists $t \in R_{P}$ such that $t\left(z_{1}\right)=0$ and $t\left(z_{2}\right)=s\left(z_{2}\right)$. As $t \in J_{z_{1}} R_{P}$ and $s-t \in J_{z_{2}} R_{P}$, we have $s \in J_{z_{1}} R_{P}+J_{z_{2}} R_{P}$. This implies $R_{P}=J_{z_{1}} R_{P}+J_{z_{2}} R_{P}$, a contradiction because $J_{z_{1}}, J_{z_{2}}$ are contained in $P$. Hence $Y$ has a unique maximal element.

We have proved that $\mathfrak{A}_{P}$ is a sharp flasque sheaf of $C M$ local $F$-algebras on a ranked closed subposet $Y$ with a unique maximal element. To prove (i) $\Rightarrow$ (ii) in the theorem, it suffices to prove that, for every $z \in X, \bar{X}^{z}$ is $F$-spherical and $X^{z}$ is $F$-acyclic if $\operatorname{bight}\left(J_{z}\right)>\mathrm{rk}(z)-1$. Let us choose a minimal prime ideal $P$ of $J_{z}$ such that $\operatorname{ht}(P)=\operatorname{bight}\left(J_{z}\right)$. Localizing $\mathfrak{A}$ at $P$, we obtain a sharp flasque sheaf $\mathfrak{A}_{P}$ of $C M$ local $F$-algebras on a ranked closed subposet $Y$ with a unique maximal element. The section ring is the $C M$ local ring $R_{P}$. Note that the sharp assumption implies that $P$ cannot contain $J_{x}$ for every $x \in X$ and $x>z$. Thus $z$ is the unique maximal element of $Y$. So $Y=\bar{X}^{z}$. Since $\operatorname{ht}\left(J_{z} R_{P}\right)=\operatorname{bight}\left(J_{z}\right)$, we deduce that $\operatorname{bight}\left(J_{z}\right)>\operatorname{rk}(z)-1$ if and only if $h t\left(J_{z} R_{P}\right)>\operatorname{rk}(z)-1$. Hence we can reduce the proof of (i) $\Rightarrow$ (ii) to the case that $\mathfrak{A}$ is a sharp flasque sheaf of $C M$ local $F$-algebras on a ranked poset $X$ with a unique maximal element, and the section ring is a $C M$ local ring.

Moreover, to prove (ii) $\Rightarrow$ (i) in the theorem, it suffices to prove that $R_{M}$ is $C M$ for every maximal ideal $M$ of $R$. Let $M$ be a maximal ideal of $R$. Localizing $\mathfrak{A}$ at $M$, we obtain a sharp flasque sheaf $\mathfrak{A}_{M}$ of $C M$ local $F$-algebras on a ranked closed subposet $Y$, and $Y$ has a unique maximal element. Note that if $X$ is $F$-spherical, 
then $Y$ is also $F$-spherical. Furthermore, $\operatorname{bight}\left(J_{z} R_{M}\right) \leq \operatorname{bight}\left(J_{z}\right)$ for $z \in Y$. This implies that $\operatorname{bight}\left(J_{z}\right)>\operatorname{rk}(z)-1$ for $z$ in $Y$ if $\operatorname{bight}\left(J_{z} R_{M}\right)>\operatorname{rk}(z)-1$. Therefore the condition (ii) still holds for $\mathfrak{A}_{P}$ on $Y$. Thus we can also reduce the proof of (ii) $\Rightarrow$ (i) to the case that $\mathfrak{A}$ is a sharp flasque sheaf of $C M$ local $F$-algebras on a ranked poset $X$ with a unique maximal element.

So, in the following proof we assume that $\mathfrak{A}=\left(A_{x}, \rho_{y x}\right)$ is a sharp flasque sheaf of $C M$ local $F$-algebras on a ranked poset $X$ with a unique maximal element $m$, and the section ring of $\mathfrak{A}$ is a local ring $R$ with the unique maximal ideal $M$. Put $n=\operatorname{rk}(X)$ and $d=\operatorname{dim} R$.

(i) $\Rightarrow$ (ii). Clearly, $\operatorname{dim} A_{x}+\mathrm{ht} J_{x}=d$ and $\operatorname{bight}\left(J_{x}\right)=$ ht $J_{x}$, for every $x \in X$, because $A_{x}$ and $R$ are $C M$. We use induction on $n$ to prove the conclusion.

For $n=1$, the result is trivial. For $n=2$, it is clear that $X$ is $F$-spherical. It remains to prove $X^{x}$ is $F$-acyclic if $\operatorname{ht}\left(J_{x}\right)>\operatorname{rk}(x)-1$. According to Proposition $1.2 \operatorname{ht}\left(J_{x}\right)=0$ for every $x \neq m$. It suffices to prove that $X^{m}$ is $F$-acyclic if ht $\left(J_{m}\right)>1$. Suppose on the contrary that $X^{m}$ is not connected. So by Proposition 2.3 , depth $J_{m} \leq 1$. Thus ht $J_{m} \leq 1$ because $R$ is $C M$, a contradiction.

Now, suppose the conclusion holds for those posets with rank less than $n(n \geq 3)$. Let us consider the case of $X$ with $\operatorname{rk}(X)=n$. For every $x \in X(x \neq m)$, as we have seen in the above, the localization $\mathfrak{A}_{P_{x}}$ of $\mathfrak{A}$ at $P_{x}$ is a sharp flasque sheaf of $C M$ local $F$-algebras on $\bar{X}^{x}$ with the section ring $R_{P_{x}}$. Since $\operatorname{rk}\left(\bar{X}^{x}\right) \leq n-1$, by the induction hypothesis, we conclude that $\bar{X}^{x}$ is $F$-spherical and $X^{x}$ is $F$-acyclic if ht $J_{x}>$ rk $(x)-1$. This implies that $X^{m}$ is $F$-spherical and $X^{x}$ is $F$-acyclic for every $x \in X^{m}$ with $\operatorname{ht}\left(J_{x}\right)>\operatorname{rk}(x)-1$.

By Lemma 2.1, the sheaf cohomology groups $\mathrm{H}^{i}\left(X^{m}, \mathfrak{A} \mid X^{m}\right)$ can be computed as homology of the complex

$$
0 \rightarrow C^{0} \rightarrow C^{1} \rightarrow C^{2} \rightarrow \cdots \rightarrow C^{n-2} \rightarrow 0 .
$$

It follows from the remark in Section 2 that each $C^{i}(0 \leq i \leq n-2)$ is the direct sum of some $A_{x}$ with $\operatorname{rk}(x)=i+1$ and $\operatorname{ht}\left(J_{x}\right)=\operatorname{rk}(x)-1$. According to Proposition 1.3, there exists an $R$-sequence $r_{1}, \cdots, r_{n-1}$ such that $r_{1}(x)=r_{2}(x)=\cdots=r_{i}(x)=0$ and $r_{i+1}(x), \cdots, r_{n-1}(x)$ is an $A_{x}$-sequence for $x \in X$ with $\operatorname{rk}(x)=i+1$ and $\operatorname{ht}\left(J_{x}\right)=\operatorname{rk}(x)-1$. Hence the sequence $r_{i}, r_{i+1}, \cdots, r_{n-1}$ is a $C^{i}$-sequence.

By Proposition 4.1 in [8], we have isomorphisms

$$
\mathrm{H}^{i}\left(X^{m}, \mathfrak{A} \mid X^{m}\right) \simeq \widetilde{\mathrm{H}}^{i}\left(X^{m}, A_{m}\right) \text { for } i>0
$$

and the short exact sequence

$$
0 \rightarrow R \rightarrow \mathrm{H}^{0}\left(X^{m}, \mathfrak{A} \mid X^{m}\right) \rightarrow \widetilde{\mathrm{H}}^{0}\left(X^{m}, A_{m}\right) \rightarrow 0 .
$$

Since $\operatorname{rk}(m) \geq 3$, it follows from the sharp assumption on $\mathfrak{A}$ that ht $\left(J_{m}\right) \geq 2$. Thus $\operatorname{depth}\left(J_{m}\right) \geq 2$ because $R$ is $C M$. By Proposition $2.3, X^{m}$ is connected. This implies that $\widetilde{\mathrm{H}}^{0}\left(X^{m}, A_{m}\right)=0$. So $\mathrm{H}^{0}\left(X^{m}, \mathfrak{A} \mid X^{m}\right)=R$, that is, we have the following augmented complex of (2.5):

$$
0 \rightarrow R \rightarrow C^{0} \rightarrow C^{1} \rightarrow \cdots \rightarrow C^{n-2} \rightarrow 0 .
$$

To prove that $X$ is $F$-spherical, it sufficies to prove $\widetilde{\mathrm{H}}^{i}\left(X^{m}, F\right)=0$ for $0 \leq$ $i \leq n-3$. Suppose $\widetilde{\mathrm{H}}^{i}\left(X^{m}, F\right) \neq 0$ for some $i(0 \leq i \leq n-3)$. As we have proved $\widetilde{\mathrm{H}}^{0}\left(X^{m}, F\right)=0$, we have $n \geq 4$. By the Universal Coefficient Theorem, we 
have that not all groups $\widetilde{\mathrm{H}}^{i}\left(X^{m}, A_{m}\right)(1 \leq i \leq n-3)$ are trivial. Thus, by $(2.5)$, not all groups $\mathrm{H}^{i}\left(X^{m}, \mathfrak{A} \mid X^{m}\right)=0(1 \leq i \leq n-3)$. We will prove that this is impossible by showing that $r_{1}, r_{2}, \cdots, r_{k+2}$ is not $R$-regular. The method is similar to a discussion of Yuzvinsky in the proof of Theorem 5.1 in [8].

Let us consider the sequence (2.7). Put $Z^{i}=\operatorname{Ker}\left(C^{i} \rightarrow C^{i+1}\right)$ and $B^{i}=$ $\operatorname{Im}\left(C^{i-1} \rightarrow C^{i}\right)(0 \leq i \leq n-2)$; here we use the convention $C^{-1}=R$. We will use another convention in the following by setting the boundary homomorphisms of (2.6) equal to $\partial$. Let $c \in Z^{k} \backslash B^{k}$. We define recursively a triangular matrix with entries $a_{p q} \in C^{P-1}, q=p+1, \cdots, k+2, p=0,1, \cdots, k+1$, subject to the relations

$$
\begin{gathered}
\sum_{q=p+1}^{k+2} r_{q}^{2} \partial a_{p q}=0, \quad p=0,1, \cdots, k+1, \\
\partial a_{p q}=-r_{p+1}^{2} a_{p+1, q}, \quad q=p+2, \cdots, k+2, p=0,1, \cdots, k+1 .
\end{gathered}
$$

For $p=k+1$, we put $a_{k+1, k+2}=c$. It is clear that $a_{k+1, k+2}$ satisfies (2.8). Suppose that $q<k+1$ and a system $a_{p+1, p+2}, \cdots, a_{p+1, k+2}$ is already defined satisfying (2.9). Since $r_{p+1} \in \bigcap_{\mathrm{rk}(x)=p+2} J_{x}$, we have $r_{p+1} C^{p} \subseteq Z^{p}$. Moreover,

$$
r_{p+1}^{2} C^{p} \subseteq B^{p} .
$$

If $p<k$, this follows from the exactness of (2.7) at $C^{p}$. For $p=k, Z^{k} / B^{k} \simeq$ $\widetilde{\mathrm{H}}^{k}\left(X^{m}, A_{m}\right)$ and $r_{p+1} \in J_{m}$. So $r_{p+1} Z^{k} \subseteq B^{k}$. This implies that $r_{p+1}^{2} C^{p} \subseteq B^{p}$. Hence, we can choose $a_{p q} \in C^{p-1}(q=p+2, \cdots, k+2)$ satisfying (2.9).

In order to define $a_{p, p+1}$ we observe that $b=\sum_{q=p+2}^{k+2} r_{q}^{2} a_{p+1, q} \in Z^{p}$. Furthermore, we observe that $b \in B^{p}$. If $r<k$, this follows from the exactness of (2.7) at $C^{p}$; If $p=k$, we have $b=r_{k+2}^{2} c$. Since $c \in Z^{k}$, we have $r_{k+2}^{2} c \in B^{p}$. Hence we can can choose $a_{p, p+1} \in C^{p-1}$ satisfying $\partial a_{p, p+1}=b$. One easily checks that

$$
a_{p q}(p=0,1, \cdots, k+1, q=p+1, \cdots, k+2) \text { satisfy }(2.8) .
$$

Put $I_{t} B^{i}=\left(r_{t}^{2}, \cdots, r_{k+2}^{2}\right) B^{i}(i \leq t \leq k+1), I_{t} C^{i}=\left(r_{t}^{2}, \cdots, r_{k+1}^{2}\right) C^{i}$ and $b_{i}=\partial a_{0 i}(1 \leq i \leq k+2)$. For $p=0(2.8)$ reduces to $\sum_{i=1}^{k+2} r_{i}^{2} b_{i}=0$ or $r_{k+2}^{2} b_{k+2} \in$ $\left(r_{1}^{2}, \cdots, r_{k+1}^{2}\right) R=I_{1} B^{0}$ (identifying $B^{0}$ with $R$ ). Now, we claim that $b_{k+2} \notin I_{1} B_{0}$. Suppose on the contrary $b_{k+2}=\partial a_{0, k+2} \in I_{1} B_{0}$. Using this as the base of induction on $p$, we assume that for some $p, 0<p<k-1$, we have $\partial a_{p, k+2} \in I_{p+1} B^{p}$. Due to (2.9), this can be rewritten as $r_{p+1}^{2} a_{p+1, k+2} \in I_{p+1} B^{p}$, or, in other words, there exists $e \in B^{p}$ such that $r_{p+1}^{2}\left(a_{p+1, k+2}-e\right) \in I_{p+2} B^{p}$. By the fact that $r_{p+1}^{2}, \cdots, r_{k+1}^{2}$ is $C^{p}$-regular, we have that $\left(a_{p+1, k+2}-e\right) \in I_{p+2} C^{p}$. This implies that $\partial\left(a_{p+1, k+2}\right) \in I_{p+2} C^{p}$. In particular, for $p=k-1$ we have $r_{k+1}^{2} c=-\partial a_{k, k+2} \in$ $r_{k+1}^{2} B^{k}$. Since $r_{k+1}^{2}$ is $C^{k}$-regular, it follows that $c \in B^{k}$, which is a contradiction because $c \notin B^{k}$. Thus $b_{k+2} \notin\left(r_{1}^{2}, \cdots, r_{k+1}^{2}\right) R$. Since $r_{1}^{2}, \cdots, r_{k+2}^{2}$ is an $R$-sequence and $r_{k+2}^{2} b_{k+2} \in\left(r_{1}^{2}, \cdots, r_{k+1}^{2}\right) R$, we have $b_{k+2} \in\left(r_{1}^{2}, \cdots, r_{k+1}^{2}\right) R$, a contradiction. Therefore, $X$ must be $F$-spherical.

Now, it remains to prove that $X^{m}$ is acyclic if ht $J_{m}>n-1$. Suppose this conclusion is false, that is, ht $P_{m}>n-1$ and $X^{m}$ is not acyclic. Since $X$ is $F$-spherical and $\mathfrak{A}$ is flasque, the complex

$$
0 \longrightarrow R \longrightarrow C^{0} \stackrel{\partial_{0}}{\longrightarrow} C^{1} \longrightarrow \cdots \longrightarrow C^{n-2} \stackrel{\partial_{n-2}}{\longrightarrow} C^{n-1} \longrightarrow 0
$$


from Lemma 2.1 is acyclic. Observe that $\operatorname{ht}\left(J_{x}\right) \geq \operatorname{rk}(x)-1$ for every $x \in X$, and $X^{x}$ is $F$-acyclic if ht $J_{x}>\operatorname{rk}(x)-1(x \neq m)$. Thus each $C^{i}$ is either a $C M$ $R$-module of dimension $d-i$ or a zero $R$-module for each $i(0 \leqslant i \leqslant n-2)$ by the induction hypothesis. The above hypothesis means that $C^{n-1} \neq 0$. Put $Z^{i}=$ Ker $\partial_{i}(1 \leqslant i \leqslant n-2)$. Let us consider the following short exact sequences:

$$
\begin{aligned}
0 & \rightarrow R \rightarrow C^{0} \rightarrow Z^{1} \rightarrow 0 \\
0 & \rightarrow Z^{1} \rightarrow C^{1} \rightarrow Z^{2} \rightarrow 0 \\
& \quad \cdots \quad \quad \cdots \\
0 & \rightarrow Z^{n-2} \rightarrow C^{n-2} \rightarrow C^{n-1} \rightarrow 0 .
\end{aligned}
$$

We assert that $C^{i} \neq 0$ and $\operatorname{dim} Z^{i}=d-i \quad(1 \leqslant i \leqslant n-2)$. In fact, from the sharp assumption on $\mathfrak{A}$, we have $\operatorname{dim} C^{n-2}>\operatorname{dim} C^{n-1}$. This implies $C^{n-2} \neq 0$ and $\operatorname{dim} C^{n-2}=d-n+2$. Consider the last short exact sequence in (2.10); we have $\operatorname{dim} Z^{n-2}=d-n+2$ because $\operatorname{dim} C^{n-2}=\max \left\{\operatorname{dim} Z^{n-2}, \operatorname{dim} C^{n-1}\right\}$. Similarly, we can prove $C^{n-3}, \cdots, C^{1}$ are all nonzero modules and $\operatorname{dim} Z^{i}=d-i(1 \leqslant i \leqslant n-3)$. Since $R, C^{0}$ are $C M$, by applying the local cohomology functors $\left\{\mathrm{H}_{M}^{i}(-)\right\}_{i \geqslant 0}$ to the first short exact sequence in (2.8), we obtain the following long exact sequence:

$$
\cdots \rightarrow \mathrm{H}_{M}^{i-1}\left(Z^{1}\right) \rightarrow \mathrm{H}_{M}^{i}(R) \rightarrow \mathrm{H}_{M}^{i}\left(C^{0}\right) \rightarrow \mathrm{H}_{M}^{i}\left(Z^{1}\right) \rightarrow \cdots .
$$

As $R, C^{0}$ are $C M$ modules of dimension $d$, we have $\mathrm{H}_{M}^{i}(R)=0, \mathrm{H}_{M}^{i}\left(C^{0}\right)=0$ for $i<d$. So $\mathrm{H}_{M}^{i}\left(Z^{1}\right)=0$ for $i<d-1$. This implies that $Z^{1}$ is $C M$ because $\operatorname{dim} Z^{1}=d-1$. Continue the processes, we can prove that $Z^{2}, \cdots, Z^{n-2}$ are $C M$. Now, let us consider the long exact sequence of local cohomology derived from the last short exact sequence in (2.10):

$$
\cdots \rightarrow \mathrm{H}_{M}^{i}\left(Z^{n-2}\right) \rightarrow \mathrm{H}_{M}^{i}\left(C^{n-2}\right) \rightarrow \mathrm{H}_{M}^{i}\left(C^{n-1}\right) \rightarrow \cdots .
$$

Since $\mathrm{H}_{M}^{i}\left(Z^{n-2}\right)=0$ and $\mathrm{H}_{M}^{i}\left(C^{n-2}\right)=0$ for $i<d-n+2$, we have $\mathrm{H}_{M}^{i}\left(C^{n-1}\right)=0$ for $i<d-n+1$. Note that $C^{n-1}$ is a nonzero $C M$ module and $\operatorname{dim} C^{n-1}<d-n+2$. So $\operatorname{dim} C^{n-1}=d-n+1$, i.e. ht $P_{m}=n-1$, a contradiction. Hence $X^{m}$ is acyclic for ht $P_{m}>n-1$.

(ii) $\Rightarrow$ (i). First, we claim that $\operatorname{dim} A_{x}=d$ for every $x \in X_{\min }$. We use induction on $n$. If $n=1$, there is nothing to prove. For $n=2$, suppose there exist $x, y \in X_{\min }$ such that $\operatorname{dim} A_{x}<\operatorname{dim} A_{y}$. This implies $\operatorname{dim} A_{m}<\operatorname{dim} A_{x}<\operatorname{dim} A_{y}$, by the sharpness hypothesis on $\mathfrak{A}$. Hence $\operatorname{ht}\left(J_{m} / J_{y}\right)=\operatorname{dim} A_{y}-\operatorname{dim} A_{m} \geq 2$. It follows that ht $J_{m} \geq 2$. By the assumption (ii), $X^{m}$ is $F$-acyclic, which is a contradiction. So our claim is true for $\operatorname{rk}(X)=2$.

For $n>2$, put $X_{1}=\left\{x \in X_{\min } \mid \operatorname{dim} A_{x}=d\right\}$. It follows from Proposition 1.2 that $X_{1} \neq \emptyset$. Suppose our claim is false, that is, $X_{1} \neq X_{\min }$. Since $X$ is $F$-spherical and $\operatorname{rk}\left(X^{m}\right) \geq 2$, we have $\widetilde{\mathrm{H}}^{0}\left(X^{m}, F\right)=0$. This implies that $X^{m}$ is connected. Hence we can choose an element $z \in X$ with $\operatorname{rk}(z)=n-1$ such that $z>x$ and $z>y$ for some $x \in X_{1}$ and $y \in X_{\min } \backslash X_{1}$. Let $P$ be a minimal prime ideal of $J_{z}$. Localizing the sheaf $\mathfrak{A}$ at $P$, we obtain a sharp flasque sheaf of $C M$ local $F$-algebras $\mathfrak{A}_{P}$ on $\bar{X}^{z}$ which still satisfies the condition (ii). So by induction $\operatorname{dim} R_{P} / J_{x} R_{P}=\operatorname{dim} R_{P} / J_{y} R_{P}$. As $A_{x}$ and $A_{y}$ are $C M$ local rings, we assert that $\operatorname{dim} A_{x}=\operatorname{dim} A_{y}$. This contradicts the choices of $x$ and $y$. So $X_{1}=X_{\min }$, and that ends the proof of our claim.

By the claim and Proposition 1.2, one can see easily that every maximal chain of primes of $R$ has length $d$, because each $A_{x}\left(x \in X_{\min }\right)$ is $C M$. Hence we 
conclude that for every $x \in X$, $\operatorname{bight}\left(J_{x}\right)=\operatorname{ht}\left(J_{x}\right)$ and $\operatorname{dim} A_{x}=d-\operatorname{ht}\left(J_{x}\right)$. As $X$ is $F$-spherical and $\mathfrak{A}$ is flasque, by Lemma (2.1), we have the following long exact sequence:

$$
0 \rightarrow R \rightarrow C^{0} \stackrel{\partial_{0}}{\rightarrow} \cdots \stackrel{\partial_{n-2}}{\rightarrow} C^{n-1} \rightarrow 0
$$

By the $F$-acyclicity of $X^{x}$ for every $x \in X$ with ht $J_{x}>\operatorname{rk}(x)-1$ and the obvious fact that ht $J_{z} \geq \operatorname{rk}(z)-1$ for every $z \in X$, we assert that each $C^{i}(0 \leq i \leq n-1)$ is either a $C M R$-module of dimension $d-i$ or a zero $R$-module.

Put $Z^{i}=\operatorname{Ker} \partial_{i}(0 \leqslant i \leqslant n-2)$ and $Z^{n-1}=C^{n-1}$. It is clear that $Z^{0} \simeq R$. Let us consider the following short exact sequences:

$$
\begin{aligned}
0 & \rightarrow Z^{0} \rightarrow C^{0} \rightarrow Z^{1} \rightarrow 0 \\
0 & \rightarrow Z^{1} \rightarrow C^{1} \rightarrow Z^{2} \rightarrow 0 \\
& \quad \cdots \\
& \cdots \\
0 & \rightarrow Z^{n-2} \rightarrow C^{n-2} \rightarrow C^{n-1} \rightarrow 0 .
\end{aligned}
$$

If for some $i(1 \leq i \leq n-1), C^{i}=0$, we have $C^{j}=0$ for $j>i$ because either $\operatorname{dim} C^{j}=d-j$ or $C^{j}=0$. So without loss of generality, we can assume $C^{i} \neq$ 0 for all $i(0 \leqslant i \leqslant n-1)$. For each $i(0 \leqslant i \leqslant n-2)$, we have $\operatorname{dim} C^{i}=$ $\max \left\{\operatorname{dim} Z^{i}, \operatorname{dim} Z^{i+1}\right\}$. Thus $\operatorname{dim} C^{i}=\operatorname{dim} Z^{i}=d-i$ because $\operatorname{dim} C^{i}>\operatorname{dim} C^{i+1}$ and $\operatorname{dim} Z^{i+1} \leq \operatorname{dim} C^{i+1}$. Applying the local cohomology functors $\left\{\mathrm{H}_{M}^{i}(-)\right\}_{i \geqslant 0}$ to the last short exact sequence in $(2.11)$, we derive the long exact sequence

$$
\cdots \rightarrow \mathrm{H}_{M}^{i}\left(Z^{n-2}\right) \rightarrow \mathrm{H}_{M}^{i}\left(C^{n-2}\right) \rightarrow \mathrm{H}_{M}^{i}\left(C^{n-1}\right) \rightarrow \mathrm{H}_{M}^{i+1}\left(Z^{n-2}\right) \rightarrow \cdots .
$$

Observe that $\mathrm{H}_{M}^{i}\left(C^{n-2}\right)=0$ for $i<d-n+2$ and $\mathrm{H}_{M}^{i}\left(C^{n-1}\right)=0$ for $i<d-n+1$. Hence $\mathrm{H}_{M}^{i}\left(Z^{n-2}\right)=0$ for $i<d-n+2$. So $Z^{n-2}$ is $C M$. Continue the processes along with other short exact sequences in (2.11), we can prove $Z^{n-2}, \cdots, Z^{1}, Z^{0}$ are all $C M$. Therefore $R$ is $C M$.

Remark. There is a gap in the proof of the Theorem 3.3 of the paper [8], since $J(i)=\bigcap_{\mathrm{rk}(x)=i} J_{x}$ may not be a unmixed ideal of $R$, i.e. the heights of the minimal prime ideals of $J(i)$ may not be the same. So a maximal $R$-sequence in $J(i)$ may not be a maximal $R$-sequence in every minimal prime ideal of $J(i)$. Thus $r_{1}, \ldots, r_{n+d-1}$ in the proof of Theorem 3.3 of [8] may not be a standard system. Hence even in the case that $A_{x}(x \in X)$ are all $C M$ local domains, the $F$-spherical properties in (i) $\Rightarrow$ (ii) of our Theorem 2.4 cannot be deduced directly from the results in [8].

There are two interesting corollaries derived directly from Theorem 2.4.

Corollary 2.12. Let $\mathfrak{A}, X$ and $R$ be as in Theorem 2.4, and let $R$ be CM. If $X^{y}$ is not $F$-acyclic, then bight $\left(J_{x}\right)=\mathrm{rk}(x)-1$ for all $x \in X$ with $x<y$.

Corollary 2.13. Let $\mathfrak{A}, X$ and $R$ be as in Theorem 2.4, and let $R$ be $C M$. If bight $J_{x}>$ rk $(x)-1$ for some $x \in X$, then $X^{y}$ is $F$-acyclic for $y>x$.

Before we end the paper, we give a simple application of the main result, Theorem 2.4. Let $P=\{x, y, u, v\}$ be a poset with the relations

$$
u<x, \quad v<x, \quad u<y, \quad v<y .
$$

Let $X, Y$ be two indeterminants and $\mathfrak{A}$ be a sheaf on $P$ with stalks:

$$
A_{u}=F[X], \quad A_{v}=F[Y], \quad A_{x}=F[X] /\left(X^{2}-1\right), \quad A_{y}=F
$$


and with homomorphisms

$$
\begin{gathered}
\rho_{x u}(f(X))=f(X) \bmod \left(X^{2}-1\right), \quad \rho_{y u}(f(X))=f(0), \\
\rho_{x v}(f(Y))=f(X) \bmod \left(X^{2}-1\right), \quad \rho_{y v}(f(Y))=f(0) .
\end{gathered}
$$

It is easy to check that $\mathfrak{A}$ is a sharp flasqe sheaf of $C M F$-algebras on $P$. Thus by Theorem 2.4 the section ring $R=\Gamma(\mathfrak{A})$ is $C M$.

\section{ACKNOWLEDGMENT}

The author wishes to express his gratitude to the referee for helpful suggestions and comments.

\section{REFERENCES}

[1] M. Auslander, D. A. Buchsbaum, Codimension and multiplicity, Ann. of Math. 68 (1958), 625-657. MR 20:6414

[2] K. Baclawski, Whitney numbers of geometric lattice, Adv. in Math. 16 (1975), 125-138. MR 52:7933

[3] K. Baclawski, Cohen-Macaulay ordered sets, J. Alg. 63 (1980), 226-258. MR 81m:06002

[4] R. Godement, Topologie algébrique et théorie des faisceaux, Hermann et cie, Paris, 1958. MR 21:1583

[5] H. Matsumura, Commutative Algebra, Benjamin Reading, MA, 1980. MR 82i:13003

[6] G. Reisner, Cohen-Macaulay quotients of polynomial rings, Adv. in Math. 21 (1976), 30-49. MR 53:10819

[7] R. Stanley, Combinatorics and Commutative Algebra, Birkhäuser, Boston, 1983. MR 85b:05002

[8] S. Yuzivinsky, Cohen-Macaulay rings of sections, Adv. in Math. 63 (1987), 172-195. MR 88d:13039

[9] S. Yuzivinsky, Flasque sheaves on posets and Cohen-Macaulay unions of regular varieties, Adv. in Math. 73 (1989), 24-42. MR 90a:13038

Department of Mathematics, Shanghai Normal University, Shanghai 200234, China

E-mail address: shtunet@public.sta.net.cn 